\title{
A ANTA (Tapirus terrestris) EM FRAGMENTOS DE FLORESTA COM ARAUCÁRIA, PARANÁ, BRASIL
}

\author{
Gisley Paula Vidolin ${ }^{1}$, Daniela Biondi ${ }^{2}$, Adilson Wandembruck ${ }^{3}$ \\ ${ }^{1}$ Bióloga, Dra ${ }^{\mathrm{a}}$, Bio Situ Projetos e Estudos Ambientais Ltda., Piraquara, Paraná, Brasil - paula@ biositu.com.br \\ ${ }^{2}$ Eng $^{\mathrm{a}}$. Florestal, Dra ${ }^{\mathrm{a}}$, Depto. de Ciências Florestais, UFPR, Curitiba, PR, Brasil - dbiondi@ufpr.br \\ ${ }^{3}$ Eng. Florestal, Bio Situ Projetos e Estudos Ambientais Ltda., Piraquara, Paraná, Brasil - adilson@ biositu.com.br \\ Recebido para publicação: 08/02/2010 - Aceito para publicação: 12/06/2011
}

\begin{abstract}
Resumo
Este estudo teve como objetivo conhecer o tamanho e área de uso, abundância relativa e distribuição espacial da anta no Corredor Ecológico Araucária. Para tanto, a pesquisa foi conduzida em 14 propriedades localizadas em um recorte espacial de $659 \mathrm{~km}^{2}$, situado entre as coordenadas geográficas $26^{\circ} 26^{\prime} 35^{\prime \prime}$ de latitude S, $51^{\circ} 46^{\prime} 10^{\prime \prime}$ de longitude W e $26^{\circ} 12^{\prime} 19^{\prime \prime}$ de latitude S, $51^{\circ} 24^{\prime} 45^{\prime \prime}$ de longitude $\mathrm{W}$. O método de amostragem utilizado consistiu no mapeamento dos pontos de ocorrência da anta, tendo como base entrevista com moradores locais e a busca por indícios, como pegadas, carreiros e outros sinais que atestassem a sua presença nas áreas amostrais. As áreas foram visitadas mensalmente ao longo do período de 18 meses, de janeiro de 2007 a julho de 2008, e todos os pontos (UTM) onde foram encontrados sinais da espécie foram marcados em GPS e, posteriormente, plotados em mapa. Os pontos extremos foram unidos, resultando em um polígono de área de uso dos animais, o qual teve sua área calculada no programa ArcMap versão 9.2. O índice de abundância relativa foi estimado com base em todos os eventos em que a espécie foi visualizada e que os animais puderam ser contabilizados, considerando-se os registros individualizados para cada propriedade. Obteve-se a estimativa de uma média de quatro indivíduos para a região numa área de 4.314 ha ou $43 \mathrm{~km}^{2}$. Essa densidade relativa obtida pode ser considerada baixa. Possivelmente, a caça seja a principal causa do declínio populacional da espécie na região estudada.

Palavras-chave: Corredor ecológico; paisagem; ecologia da paisagem.
\end{abstract}

\begin{abstract}
The tapir (Tapirus terrestris) in forest fragments with Araucária in Paraná, Brazil. This study aimed to investigate the using area for use of tapir (Tapirus terrestris) along the Araucaria Ecological Corridor in relation to abundance, spatial distribution and area size. The research was carried out on 14 properties located on a spatial section of $659 \mathrm{~km}^{2}$ situated between the coordinates of $26^{\circ} 26^{\prime} 35^{\prime \prime}$ latitude S and $51^{\circ} 46^{\prime} 10^{\prime \prime}$ longitude $\mathrm{W}$, and $26^{\circ} 12^{\prime} 19^{\prime \prime}$ latitude S and $51^{\circ} 24^{\prime} 45^{\prime \prime}$ longitude W. The points of occurrence of tapir were mapped based on interviews with local inhabitants and on tracks, paths, and other signs of its presence in the sampling areas. The areas were visited monthly during a period of 18 months. The points (UTM) where signs of the species were found had been marked by GPS and plotted on the map. The index of relative abundance was estimated based on all the events in which the specie was seen. Considering the values of relative abundance index for both groups of tapir studied, it was estimated an average of four individuals for an area of 4.314 ha or $43 \mathrm{~km}^{2}$. The relative density obtained was considered low. It is possible that hunting is the main cause of the population decline of the species in the studied region.

Keywords: Ecological corridor; landscape; landscape ecology.
\end{abstract}

\section{INTRODUÇÃO}

A anta (Tapirus terrestris) é o maior mamífero terrestre ocorrente no Brasil e seus aspectos biológicos são inconfundíveis, tanto por seu tamanho quanto por sua forma corporal. Devido ao seu pequeno potencial reprodutivo, longo período de gestação e longevidade e ao estilo de vida individualista, ocorrem em baixa densidade populacional, sendo vulneráveis a extinções locais, devido a variações demográficas, ambientais e perdas de diversidade genética (MEDICI et al., 2007). Paviolo et al. (2006), 
por exemplo, avaliarando o status de conservação da espécie na ecorregião da Floresta Atlântica, que contempla a província de Misiones na Argentina, sul do Brasil e leste do Paraguai, verificaram que no Paraguai a espécie subexiste em somente quatro áreas protegidas, que são a Reserva San Rafael, com 58.490 ha, a Reserva Natural de Bosque Mbaracayú, com 59.056 ha, a Itabó, com 9.885 ha, e a Reserva Biológica de Limoy, com 11.866 ha. No Brasil, nessa ecorregião, a espécie ocorre em poucos fragmentos de áreas superiores a 3.000 ha, próximos ao Parque Nacional do Iguaçu. Em Misiones, a anta está presente em áreas florestadas do centro ao norte da província (corredor verde), mas sua distribuição declinou mais de 30\% nos últimos 40 anos. Segundo esses autores, a conservação da anta nessa ecorregião depende da manutenção de grandes blocos de floresta nativa, da criação de áreas protegidas e da aplicação das leis que protegem a espécie.

A anta encontra-se listada pela União Internacional para a Conservação da Natureza (IUCN International Union for the Conservation of Nature) como "Vulnerável à Extinção" nas categorias A1cd+2c+3c (IUCN, 2008). No Brasil, apesar de não constar nas listas do IBAMA (2003 e 2008), é citada em diferentes status de ameaça em listas estaduais: em Minas Gerais e no Rio Grande do Sul consta como "criticamente em perigo", e nos estados de São Paulo, Rio de Janeiro e Paraná como "em perigo" (MIKICH; BÉRNILS, 2004). De acordo com a IUCN (2004), no Brasil existem populações consideráveis da espécie na Amazônia e no Mato Grosso do Sul, mas que, devido a diferentes tipos de pressões antrópicas, estão em declínio (PADILLA; DOWLER, 1994; REIS et al., 2006). No Paraná, ocorria em praticamente todos os biomas, no entanto, atualmente vem desaparecendo juntamente com a destruição das florestas (MIKICH; BÉRNILS, 2004). Segundo Fragoso (1994), caça é o principal fator do desaparecimento das antas em alguns locais onde sua ocorrência era comum. O desaparecimento da anta pode causar rompimento de alguns processos ecológicos, tais como predação e dispersão de sementes e de ciclos de nutrientes, os quais ajudam a manter a integridade e funcionalidade dos ambientes. Seus padrões de forrageio e de deslocamento a tornam elemento importante nas mudanças da composição e da estrutura das florestas (IUCN, 1997). Sendo assim, o direcionamento de esforços de conservação da anta no estado do Paraná é altamente justificável, já que se trata de uma espécie-chave na dinâmica florestal.

Considerando-se a hipótese de que as ações antrópicas caracterizadas pela caça e as alterações ambientais ocorrentes em áreas de Floresta Ombrófila Mista com Araucária do Paraná são os fatores responsáveis pelo declínio populacional da anta, o objetivo do trabalho foi estimar o tamanho e área de uso, abundância relativa e distribuição espacial da anta no Corredor Ecológico Araucária. Visto que não há registro de projetos de pesquisa especificamente focados na anta em áreas de Floresta Ombrófila Mista do Paraná, essas informações são muito importantes para o monitoramento ou adoção de estratégias de ações voltadas à proteção da anta e ao manejo da paisagem local.

\section{MATERIAL E MÉTODOS}

\section{Áreas de estudo}

Este estudo foi conduzido em 14 propriedades situadas na porção sul do Corredor Ecológico Araucária, criado pelo Governo do Estado do Paraná mediante o Programa Paraná Biodiversidade, em uma parceria entre o Governo de Estado e o Banco Mundial (GEF).

O Corredor Ecológico Araucária está localizado no Terceiro Planalto do estado, entre as coordenadas geográficas $50^{\circ} 55^{\prime} 39^{\prime \prime}$ e $52^{\circ} 23^{\prime} 37^{\prime \prime}$ de longitude W, e entre as latitudes S $25^{\circ} 34^{\prime} 35^{\prime \prime}$ e 26 43'28' (Figura 1). Abrange 11 municípios da região centro-sul, totalizando uma área de $12.472 \mathrm{~km}^{2}$. Faz divisas a leste com a serra da Esperança, ao sul com o estado de Santa Catarina e a oeste e norte com outros municípios paranaenses. Seu eixo principal de conexões é o rio Iguaçu e seus tributários. Além da Floresta Ombrófila Mista, há na região do Corredor a influência da Floresta Estacional Semidecidual pelo vale do rio Iguaçu, bem como de campos limpos nas regiões mais elevadas do Terceiro Planalto. Abriga diversas espécies da flora e da fauna ameaçadas de extinção (PARANÁ, 2006).

As propriedades consideradas para este estudo estão inseridas em um recorte espacial de $659 \mathrm{~km}^{2}$, situado entre as coordenadas geográficas $26^{\circ} 26^{\prime} 35^{\prime \prime}$ ' de latitude $\mathrm{S}, 51^{\circ} 46^{\prime} 10^{\prime \prime}$ ' de longitude W e $26^{\circ} 12^{\prime} 19^{\prime}$ ' de latitude S, $51^{\circ} 24^{\prime} 45^{\prime}$ ' de longitude W (Figura 2).

A Fazenda Lageado Grande (FLG), com 3.136,32 ha, de propriedade das Indústrias Pedro N. Pizzatto, está situada entre os municípios de Palmas, General Carneiro e Bituruna. O rio Iratim, que apresenta variação de largura entre 10 e $50 \mathrm{~m}$ e estende-se por cerca de $20 \mathrm{~km}$ na propriedade, é o 
principal rio da área. A porção norte da propriedade é caracterizada integralmente por floresta com predomínio de araucária (Araucaria angustifolia) e manchas de várzea. As porções central e sul da referida área são constituídas por um mosaico paisagístico de reflorestamento com pínus (Pinus spp.), floresta com predomínio de folhosas, floresta com predomínio de pinheiro e várzeas. É nessas duas porções que se concentra a grande maioria das atividades de exploração florestal.

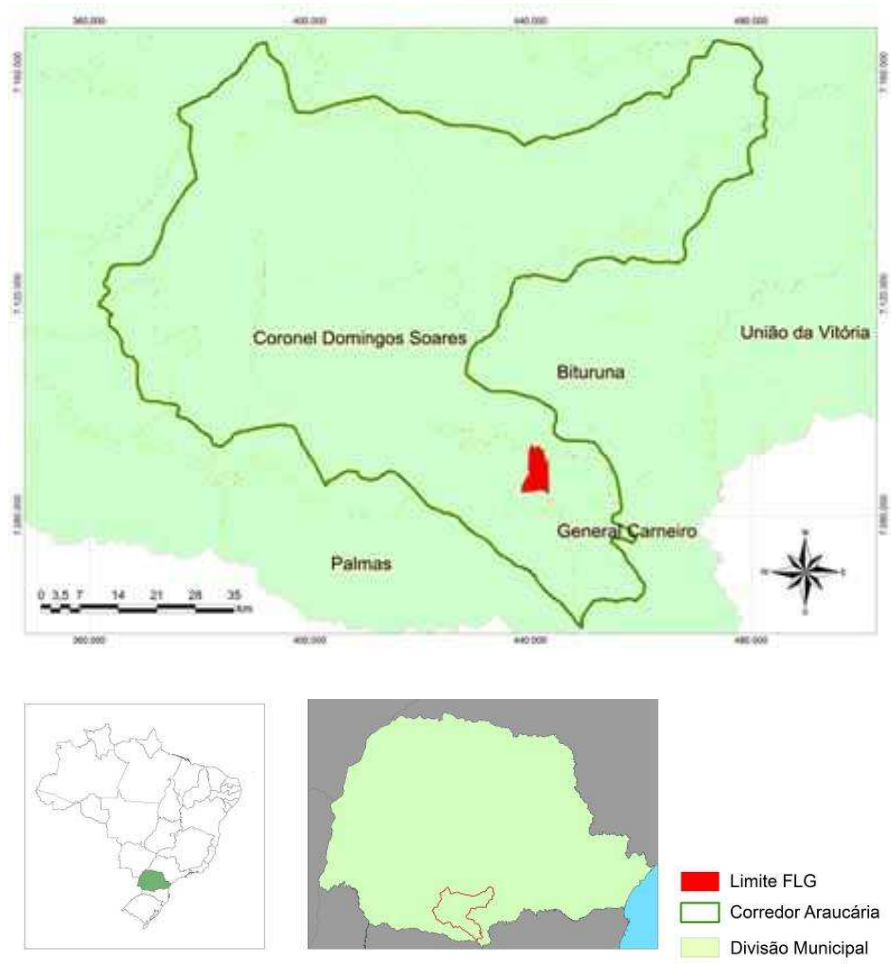

Figura 1. Mapa de localização da Fazenda Lageado Grande, Paraná, Brasil. Base de dados cartográficos: Paraná (2006).

Figure 1. Map of location of Lageado Grande Farm in Paraná, Brazil. Cartographic database: Paraná (2006).

A também chamada Fazenda Lageado Grande (cerca de 4.800 ha) e a Fazenda Santa Cruz (cerca de 2.800 ha), ambas de propriedade da Remasa Reflorestadora S.A., estão localizadas nos municípios de Bituruna e Palmas, respectivamente. São áreas constituídas por remanescentes de florestas nativas em diferentes estágios sucessionais que se alternam com extensas áreas de plantios de pínus. Os principais rios que cortam as áreas são o Lageado Grande e o São Lourenço, respectivamente.

A Fazenda Etiene (cerca de 1.500 ha), de propriedade do Sr. Ernesto Schuega, que é contígua à Fazenda Faxinal dos Santos (aproximadamente 2.500 ha), de propriedade da empresa Miguel Forte, estão ambas situadas no município de General Carneiro. Essas áreas possuem um mosaico paisagístico constituído predominantemente por áreas de floresta com predomínio de araucária em estágio avançado e médio de regeneração, intercaladas com faixas de floresta com predomínio de folhosas e várzeas. Os ambientes são semelhantes aos da FLG. O principal rio que corta a área é o Lageado Grande.

A Fazenda Santa Gema Geyer (aproximadamente 7.500 ha), de propriedade das empresas Oscar Geyer S.A., está situada entre os municípios de General Carneiro e Palmas. Essa fazenda é contígua à FLG, e assim como esta área, possui um mosaico paisagístico constituído predominantemente por grandes manchas de floresta com predomínio de araucária intercaladas com áreas de floresta com predomínio de folhosas, várzeas e reflorestamentos com pínus. O principal rio que corta a área é o Iratim.

A Fazenda São Lourenço, de propriedade do Sr. Adelar Laurides Anzileiro, está situada em Palmas. Possui um mosaico paisagístico constituído predominantemente por áreas agricultáveis e por 
reflorestamento de pínus, intercaladas com manchas de vegetação nativa, incluindo floresta com predomínio de folhosas e várzeas.

A Fazenda Santana (cerca de 2.300 ha), a Fazenda Santa Bárbara (cerca de 1.700 ha) e a Fazenda Santa Lúcia (cerca de 600 ha) são áreas contíguas situadas nos municípios de Palmas e Coronel Domingos Soares e pertencem à empresa Madepar S.A. Possuem um mosaico paisagístico constituído predominantemente por florestas com predomínio de folhosas, intercalas com manchas de vegetação secundária nas fases inicial e intermediária da sucessão vegetal. Apresentam pequenas manchas de floresta com predomínio de pinheiro e poucas manchas de várzeas.

A Fazenda Palmital, de propriedade das empresas Guararapes/Sudati S.A., situadas nos municípios de General Carneiro e Palmas, são áreas constituídas por remanescentes de florestas nativas em diferentes estágios sucessionais que se alternam com extensas áreas de plantios de pínus.

A Faxinal dos Santos e os assentamentos rurais Margens do Iratim, Paraíso do Sul e São Lourenço são caracterizados por remanescentes de florestas nativas em diferentes estágios sucessionais que se alternam com pequenas pastagens, agricultura de subsistência, áreas abandonadas e, em menor grau, por médias propriedades com agricultura mecanizada.

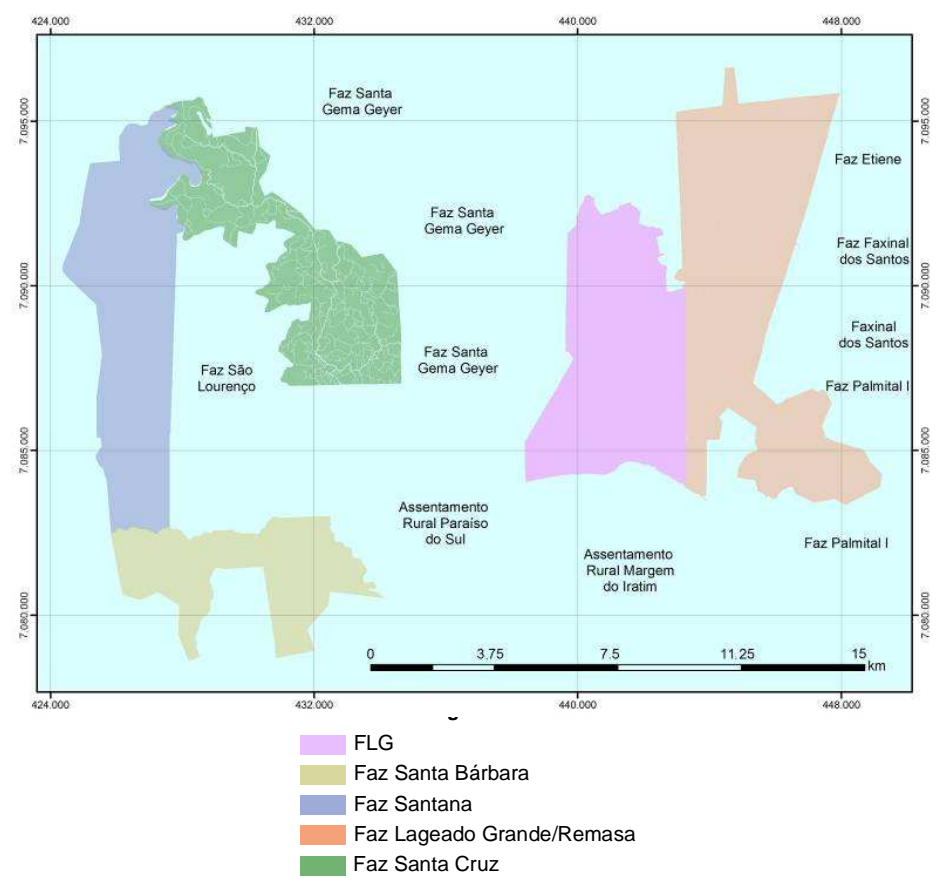

Figura 2. Mapa de localização das propriedades em macroescala. Base de dados cartográficos: Paraná (2006).

Figure 2. Map of location of the farms on a macro scale. Cartographic database: Paraná (2006).

\section{Procedimentos metodológicos}

A estimativa do tamanho da área de uso da anta foi baseada no mapeamento dos pontos de ocorrência da espécie nas 14 propriedades estudadas. As informações iniciais sobre a ocorrência desse ungulado basearam-se em entrevistas com moradores locais, que relataram seu conhecimento sobre a distribuição da espécie, bem como os trajetos frequentemente utilizados para deslocamento entre propriedades. Também foram obtidas informações sobre os principais tipos de pressões exercidas sobre a espécie, incluindo o recebimento de denúncias formais de abate dos animais. Posteriormente, foram realizadas incursões às áreas indicadas nas entrevistas, visando confirmar as informações. A constatação da ocorrência da anta nessas áreas deu-se pelo registro de sinais diagnósticos de sua presença, como pegadas, carreiros, material escatológico, vocalizações e visualizações. Essas áreas foram visitadas mensalmente ao longo do período de estudo e todos os pontos onde foram encontrados sinais da espécie 
marcados em GPS. Os registros foram plotados em um mapa de uso do solo da região (base de dados PARANÁ, 2006) e os pontos extremos foram unidos, resultando em um polígono de área de uso dos animais, a qual teve sua área calculada no programa ArcMap versão 9.2. Considerou-se como área de uso central as áreas de uso mais frequentes, e como áreas de uso total as áreas utilizadas por esse ungulado com menor frequência ou apenas em determinados períodos do ano (VIDOLIN, 2008).

Para estimar o índice de abundância relativa, considerou-se o número acumulado de registros (recentes ou frescos) obtidos para toda a extensão da área de estudo (número total de registros dividido pelo tamanho da área de estudo). O índice obtido foi extrapolado para o recorte espacial da área de vida desse tapirídeo e foi estimado o número médio de indivíduos existentes para o polígono considerado.

Segundo Gibbs (2000), a obtenção de estimativas acuradas de tamanho absoluto de populações ou suas densidades é difícil, e uma solução frequentemente usada são os índices de abundância. Caughley (1977) define esses índices de abundância relativa como um correlativo mensurável da densidade, o qual é presumivelmente relacionado com a abundância verdadeira.

\section{RESULTADOS E DISCUSSÃO}

A área de uso total da anta foi estimada em 4.314 ha $\left(\right.$ ou $\left.43 \mathrm{~km}^{2}\right)$ e a área de uso central em 3.530 ha $\left(35 \mathrm{~km}^{2}\right.$ ) (Figura 3). O mosaico paisagístico que compõe a área de uso total da espécie é constituído por $56,69 \%$ de fragmentos de floresta nativa, $27,62 \%$ de fragmentos de reflorestamento, 9,53\% de áreas de uso antrópico e 6,18\% de várzeas, as quais se encontram agrupadas em determinadas porções do polígono. Elementos de conexão formados pela vegetação ciliar dos rios representam 23,09\%.

$\mathrm{Na}$ porção leste do polígono, a anta atualmente está restrita à FLG, à Fazenda Santa Gema Geyer, de propriedade das Indústrias Oscar Geyer, e à Fazenda Lageado Grande, de propriedade da Remasa Reflorestadora S.A. Não frequenta as demais áreas inseridas nesse polígono, tais como Faxinal dos Santos, Fazenda Etiene, Fazenda Palmital 1 e 2 e Fazenda Faxinal dos Santos, áreas onde há registros históricos de sua ocorrência. Na porção oeste do polígono, sua presença é confirmada apenas para as Fazendas Santa Cruz, de propriedade da Remasa Reflorestadora S.A., Santa Bárbara e Santana, ambas de propriedade da empresa Madepar.

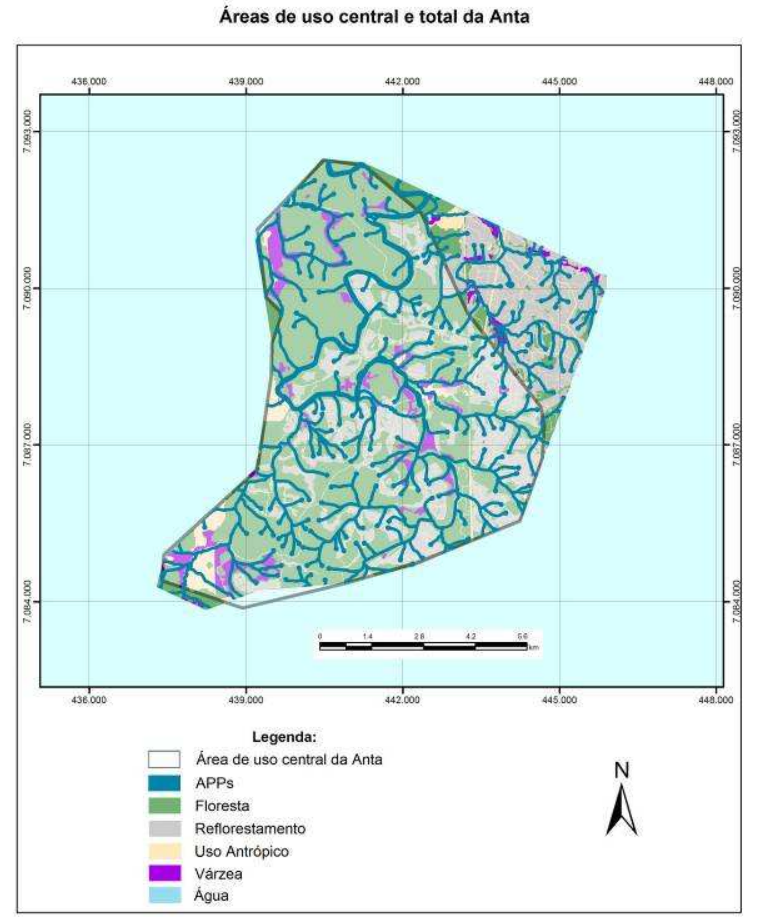

Figura 3. Áreas de uso total (polígono maior) e uso central (polígono contornado de cinza) da anta. Figure 3. Areas of total use (bigger polygon) and central use (contouring ash polygon) of the tapir.

FLORESTA, Curitiba, PR, v. 41, n. 4, p. 685 - 694, out./dez. 2011.

Vidolin, G. P.; Biondi, D.; Wandembruck, A. 
Dentro do percentual de uso central estimado em 2.351 ha (Figura 4), dos quais a FLG representa 74,96\%, a anta não utiliza ou exclui as áreas de influência antrópica direta, como, por exemplo, a vila de moradores. Tal exclusão pode estar associada à percepção da espécie em relação aos tipos de uso do solo da paisagem. Segundo Forero-Medina e Vieira (2007), fatores como mortalidade durante a dispersão e aspectos da história de vida, como tamanho do corpo e capacidade de deslocamento, podem influenciar a distribuição dos organismos em paisagens fragmentadas.

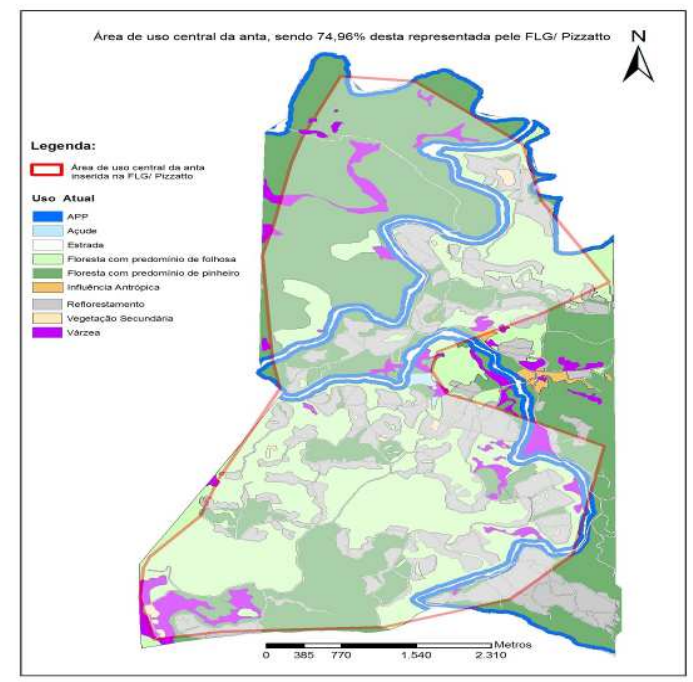

Figura 4. Tamanhos de áreas de uso central na Fazenda Lageado Grande utilizadas pela anta. Figure 4. Sizes of areas of central use in Lageado Grande Farm used by the tapir.

A anta, devido ao seu grande tamanho corporal, dificilmente passaria despercebida nas áreas próximas aos assentamentos rurais ou faxinais, onde as atividades de caça ocorrem com grande frequência. Sendo assim, a espécie exclui ou utiliza esporadicamente porções de áreas onde há maior impacto ou ameaças antrópicas. Torres et al. (2004) e Noss e Cuéller (2000) observaram uma relação importante de uso de hábitats coerente com essa inferência para a anta-centro-americana (Tapirus bairdii) no México e para a anta (Tapirus terrestris) no Chaco boliviano, com a maior abundância desse tapirídeo e todas as suas rotas de deslocamento pela paisagem sendo comumente encontradas em habitats com pouca ou nenhuma interferência antrópica. Segundo Torres et al. (2004), esse comportamento possivelmente ocorre em função de a espécie ser mais vulnerável à caça e predação em áreas abertas.

O índice de abundância relativa da anta obtido para o polígono de área de uso total foi estimado em uma média de quatro indivíduos, ou seja, 0,09 indivíduos por $\mathrm{km}^{2}$, valor bastante baixo. Outros estudos de densidade populacional com a espécie apontam valores superiores ao encontrado neste estudo, em que as estimativas refletem uma grande variação observada para diferentes ambientes. Na tabela 1 são apresentados alguns desses dados, com o objetivo de comparar os dados provenientes deste estudo com os constantes em literatura.

Conforme registros de atividades cinegéticas obtidas ao longo do estudo, pode-se verificar que na região a caça é uma atividade comumente praticada e a anta está entre uma das espécies cinegéticas procurada. Fragoso (1991) cita a caça como um dos principais fatores do desaparecimento das antas em alguns locais onde sua ocorrência era comum. Dados sobre atividades cinegéticas, levantados por Ayres e Ayres (1979) em Aripuanã (MT), por exemplo, indicaram que a anta está entre os animais mais abatidos, correspondendo a $11 \%$ do peso total do consumo de carne de animais silvestres, demonstrando a forte pressão de caça exercida sobre esse ungulado.

A baixa densidade encontrada, associada aos efeitos diretos da caça, e os aspectos reprodutivos, ecológicos e comportamentais próprios da espécie aumentam ainda mais a fragilidade da anta, como, por exemplo, seu pequeno potencial reprodutivo, nascendo um único filhote após uma gestação de treze meses, cuja maturidade sexual é atingida por volta dos dois anos. Em condições ambientais satisfatórias, os filhotes 
podem nascer a cada 14 meses, porém em habitats onde há alterações ou pressões os intervalos de nascimentos são maiores (EISENBERG, 1997). Sendo assim, a perda de indivíduos pela caça estabelece uma situação de risco ainda maior à sobrevivência e manutenção da população da anta na região estudada.

Tabela 1. Estimativas de densidade populacional da anta (Tapirus terrestris) em áreas do Pantanal, Amazônia e Mata Atlântica (baseado em MEDICI, 2010).

Table 1. Estimates of population density of the tapir (Tapirus terrestris) in areas of the Pantanal, Amazônia and Mata Attântica (based in MEDICI, 2010).

\begin{tabular}{|c|c|c|c|}
\hline Local & $\begin{array}{c}\text { Densidades } \\
\text { (ind./km²) }\end{array}$ & Método utilizado & Referências \\
\hline Fazenda Acurizal, Rio Paraguai & 0,64 & Contagem direta & Schaller (1983) \\
\hline SESC Pantanal, Barão de Melgaço & $\begin{array}{c}0,55 \\
\text { Floresta: 0,71 } \\
\text { Áreas abertas: } 0,37\end{array}$ & Transecção linear diurna & Cordeiro (2004) \\
\hline SESC Pantanal, Barão de Melgaço & $\begin{array}{l}0,30 \\
0,55 \\
1,01\end{array}$ & Transecção linear diurna & Trolle et al. (2007) \\
\hline SESC Pantanal, Barão de Melgaço & $0,58 \pm 0,11$ & Armadilhas fotográficas & Trolle et al. (2007) \\
\hline Fazenda Nhumirim, Nhecolândia & $\begin{array}{l}\quad 0,21 \\
\text { Floresta: } 0,40 \\
\text { Cerrado: } 0,13\end{array}$ & Transecção linear diurna & $\begin{array}{l}\text { Desbiez (2007) e } \\
\text { Desbiez (2009) }\end{array}$ \\
\hline Amazônia Brasileira & 0,11 a 0,52 & Transecção linear diurna & Peres (2000) \\
\hline $\begin{array}{l}\text { Centro Kayapó de Estudos Ecológicos, } \\
\text { Pará }\end{array}$ & 0,62 & Transecção linear diurna & $\underset{(2001)}{\operatorname{Zimmerman}}$ et al. \\
\hline Lago Uauaçú, Amazônia Centro-Oeste & 0,23 & Transecção linear diurna & $\begin{array}{l}\text { Haugaasen e Peres } \\
\text { (2005) }\end{array}$ \\
\hline Estação Ecológica do Maracá, Roraima & 3,3 a 3,7 & $\begin{array}{l}\text { Transecção linear diurna } \\
\text { e noturna }\end{array}$ & $\begin{array}{l}\text { Mendes-Pontes } \\
\text { (2004) }\end{array}$ \\
\hline Parque Estadual Morro do Diabo, São & 0,20 & Transecção linear diurna & Cullen et al. (2000) \\
\hline Paulo & $\begin{array}{l}0,41 \\
0,84\end{array}$ & & \\
\hline Estação Ecológica Caetetús, São Paulo & 0,47 & Transecção linear diurna & Cullen et al. (2000) \\
\hline Fazenda Mosquito, São Paulo & 0,30 & Transecção linear diurna & Cullen et al. (2000) \\
\hline Parque Estadual Mata dos Godoy, Paraná & 2,20 a 2,50 & Observações diretas/área & Rocha (2001) \\
\hline $\begin{array}{l}\text { Parque Estadual Morro do Diabo, São } \\
\text { Paulo }\end{array}$ & 0,34 & Radiotelemetria & Medici (2010) \\
\hline $\begin{array}{l}\text { Parque Estadual Morro do Diabo, São } \\
\text { Paulo }\end{array}$ & 0,64 & $\begin{array}{c}\text { Transecção linear } \\
\text { noturna }\end{array}$ & Medici (2010) \\
\hline $\begin{array}{l}\text { Parque Estadual Morro do Diabo, São } \\
\text { Paulo }\end{array}$ & 0,43 & Identificação de pegadas & Medici (2010) \\
\hline
\end{tabular}

\section{CONCLUSÕES}

- Considerando o tamanho de uso de área central da anta, a FLG assume extrema importância para a manutenção da espécie, especialmente por possuir mosaico paisagístico constituído por manchas de ambientes úmidos, como as várzeas, alternadas com ambientes florestais nativos contínuos.

- A densidade relativa obtida para a região estudada pode ser considerada baixa, sendo a caça, possivelmente, a principal causa do declínio populacional da espécie na região estudada. Cabe ressaltar que a anta é bastante sensível às alterações de ambientes e, portanto, é indicador acumulativo do estado de conservação de seus habitats. A baixa densidade relativa obtida chama a atenção para as possibilidades de essa espécie estar em "extinção ecológica", ou seja, sofreu uma redução no número de indivíduos tão drástica, que, embora continue presente na comunidade, não desempenha mais suas funções ecológicas, submetendo as áreas estudadas ao conceito de "florestas vazias" (REDFORD, 1992). Essas funções incluem a dispersão e predação de sementes, as quais são definidas por Terborgh (1988) como "estabilizadoras", pois ajudam a manter a integridade e funcionalidade dos ambientes. 
- Por esses motivos, pressões antrópicas expressivas sobre a espécie precisam urgentemente ser atenuadas, sendo as atividades de fiscalização e coibição da caça, bem como a educação ambiental da população residente, essenciais para reverter o quadro de ameaça sobre esse ungulado. O planejamento e a consolidação de estratégias de proteção, além da conscientização da população da região, são os passos iniciais para a manutenção e conservação da espécie, bem como de seus habitats.

\section{REFERÊNCIAS}

AYRES, J. M.; AYRES, C. Aspectos da caça no alto rio Aripuanã. Acta Amazônica, v. 9, n. 2, p. 287 298, 1979.

CORDEIRO, J. L. P. Estrutura e heterogeneidade da paisagem de uma unidade de conservação no nordeste do Pantanal (RPPN SESC Pantanal), Mato Grosso, Brasil: efeitos sobre a distribuição e densidade de antas (Tapirus terrestris) e de cervos-do-pantanal (Blastocerus dichotomus). 202 p. Tese (Doutorado em Ecologia) - Universidade Federal do Rio Grande do Sul, Porto Alegre, 2004.

CULLEN, L.; BODMER, R. E.; VALLADARES-PADUA, C. B. Effects of hunting in habitat fragments of the Atlantic Forests, Brazil. Biological Conservation, v. 95, p. 49 - 56, 2000.

DESBIEZ, A. L. J. Lowland tapirs in the Nhecolândia Region of the Brazilian Pantanal: population density, habitat use and threats. Tapir Conservation: The Newsletter of the IUCN/SSC Tapir Specialist Group (TSG), v. 25, p. 7 - 12, 2009.

DESBIEZ, A. L. J. Wildlife conservation in the Pantanal: hábitat alteration, invasive species and bushmeat hunting. 302 p. Tese (Doutorado) - Durrell Institute of Conservation and Ecology, University of Kent, UK, 2007.

DIRZO, R.; A. MIRANDA. Contemporary neotropical defaunation and forest structure, function, and diversity. Conservation Biology, v. 4, p. 444 - 447, 1990.

EISENBERG, J. F. Introdução. In: Tapirs - status survey and conservation action plan. IUCN/SSC Tapir Specialist Group, 1997. p. 123 - 125.

FORERO-MEDINA, G.; VIEIRA, M. V. Conectividade funcional e a importância da interação organismo-paisagem. Oecol. Bras., v. 11, n. 4, p. 493 - 502, 2007.

FRAGOSO, J. M. V. Large mammals and the community dynamics of Amazonian rains forest. 210 p. Tese (Doctor of Philosophy) - University Flórida, Flórida, 1994.

FRAGOSO, J. M. V. The effect of selective of hunting on tapirs in Belize. In: J. G. ROBINSON; K. H. REDFORD (Ed.) Neotropical wildlife use and conservation. University of Chicago Press, Chicago, USA, 1991. p. 154 - 162.

GALETTI, M. Frutos dispersos por mamíferos extintos. Ciência Hoje, Rio de Janeiro, p. 83, 2001.

GIBBS, J. P. Monitoring populations. In: BOITANI, L.; FULLER, T. K. (Ed.) Research techniques in animal ecology: controversies and consequences. New York: Columbia Inv., 2000. p. 213 - 252.

HAUGAASEN, T.; PERES, C. A. Mammal assemblage structure in Amazonian flooded and unflooded forests. Journal of Tropical Ecology, v. 21, p. 133 - 145, 2005.

INTERNATIONAL UNION FOR CONSERVATION OF NATURE AND NATURAL RESOURCES. (IUCN). Tapirs - status survey and conservation action plan. IUCN/SSC Tapir Specialist Group. 1997. $164 \mathrm{p}$.

Red list of threatened species. Disponível em: <http://www.redlist.org>. Acesso em: 15/02/2007.

Red list of threatened species: a global species assessment. Disponível em: <www.iucnred list.org>. Acesso em: 15/08/2008. 
JANZEN, D. H. Complications in interpreting the chemical defenses of trees against tropical arboreal plant eating vertebrates. The ecology of arboreal folivores, Washington, D. C., p. 73 - 84, 1978.

LIMA, J. S. Bioindicação em ecossistemas terrestres: princípios e conceitos da bioindicação. Disponível em: <www.ietec.com.br/techoje/meioambiente/tecnologias>. Acesso em: 23/10/2005.

MEDICI, E. P. Assessing the viability of lowland tapir populations in a fragmented landscape. $292 \mathrm{p}$. Tese (Doutorado) - University of Kent, Canterbury, United Kingdom, 2010.

MEDICI, E. P.; DESBIEZ, A. L. J.; GONÇALVES DA SILVA, A.; JERUSALINSKY, L.; CHASSOT, O.; MONTENEGRO, O. L.; RODRÍGUEZ, J. O.; MENDOZA, A.; QUSE, V. B.; PEDRAZA, C.; GATTI, A.; OLIVEIRA-SANTOS, L. G. R.; TORTATO, M. A.; RAMOS JR., V.; REIS, M. L.; LANDAU-REMY, G.; TAPIA, A.; MORAIS, A. A. (Ed.). Workshop para a conservação da anta brasileira: relatório final. IUCN/SSC Tapir Specialist Group \& IUCN/SSC Conservation Breeding Specialist Group, Brasil. 2007. 267 p.

MENDES-PONTES, A. R. Ecology of a community of mammals in a seasonally dry forest in Roraima, Brazilian Amazon. Mammalian Biology, v. 69, p. 319 - 336, 2004.

MIKICH, S. B.; BÉRNILS, R. S. Livro vermelho da fauna ameaçada no Estado do Paraná. Curitiba: Instituto Ambiental do Paraná, 2004.

NOSS, A. J.; CUELLER, E. Índices de abundância para fauna terrestre em El Chaco Boliviano: huellas em parcelas y em brechas barridas. In: CABRERA, E. C.; MERCOLLI, R. R. (Ed.). Manejo de fauna silvestre em Amazonia y Latinoamérica. Asunción, Paraguay, 2000. p. 73 - 82.

PADILHA, M.; DOWLER, R. C. Tapirus terrestris. Mammalian species, n. 481, p. 1 - 8, 1994.

PARANÁ. Decreto n. 3.148, de 15 de junho de 2004. Estabelece a Política Estadual de Proteção à Fauna Nativa, seus princípios, alvos, objetivos e mecanismos de execução, define o Sistema Estadual de Proteção à Fauna Nativa (SISFAUNA), cria o Conselho Estadual de Proteção à Fauna (CONFAUNA), implanta a Rede Estadual de Proteção à Fauna Nativa (Rede PRÓ-FAUNA) e dá outras providências. Diário Oficial, Curitiba, PR, 15 jun. 2004. Disponível em: <http://www.redeprofauna.pr.gov.br/modules/ conteudo/conteudo.php?conteudo=25>. Acesso em: 24/10/2011.

Avaliação ecológica rápida do Corredor Araucária. Instituto Ambiental do Paraná/ Programa Paraná Biodiversidade, 2006.

PAVIOLO, A.; DI BLANCO, Y.; VARELA, D.; DE ANGELO, C.; DI BITETTI, M. S. Regional assessment the status of lowland tapirs (Tapirus terrestris) in the Upper Paraná Atlantic Forest Ecoregion. In: INTERNACIONAL TAPIR SYMPOSIUM, 3., 2006, Buenos Aires, Argentina. Anais... Buenos Aires: IUCN, Tapir Specialist Group, Houston Zoo Naturally Wild, Temaikén, 2006.

PERES, C. A. Synergistic effects of subsistence hunting and habitat fragmentation on Amazonian Forest vertebrates. Conservation Biology, v. 15, p. 1490 - 1505, 2000.

REDFORD, K. H. A floresta vazia. In: VALLADARES PÁDUA, C.; BODMER, R. E.; CULLEN JR., L. Manejo e conservação de vida silvestre no Brasil. Brasília: CNPq; Belém: Sociedade Mamirauá, 1997, p. 1 - 22.

. The empty forest. BioScience, v. 42, n. 6, p. 412 - 422, 1992.

REIS, N. R.; PERACCHI, A. L.; PEDRO, W. A.; LIMA, I. P. Mamíferos do Brasil. Londrina, 2006. 437 p.

ROCHA, V. J. Ecologia de mamíferos de médio e grande porte do Parque Estadual Mata dos Godoy, Londrina (PR). 131 p. Tese (Doutorado em Zoologia) - Universidade Federal do Paraná, Curitiba, 2001.

SCHALLER, G. B. Mammals and their biomass on a Brasilian ranch. Arq. Zool., v. 31, n. 1, p. 1 - 36, 1983. 
SEAGLE, S. W. Generation of species-area curves by a model of animal-habitat dynamics. In: VERNER, M. L.; MORRISSON, M. L.; RALPH, C. J. Wildlife 2000 - modeling habitat relationships of terrestrial vertebrates. Madison, The University of Wisconsin, 1986. p. 281 - 286.

SOULÉ, M. E.; ALBERTS, A. C.; BOLGER, D. T. The effects of habitat fragmentation on chaparral plants and vertebrates. Oikos, v. 63, p. 39 - 47, 1992.

TAYLOR, P. D.; FAHRIG, L.; HENEIN, K.; MERRIAM, G. Connectivity is a vital element of landscape structure. Oikos, v. 68: p. 571- 573, 1993.

TERBORGH, J. The big things that run the world: a sequel to E. O. Wilson. Conser. Biol., v. 2, p. 402 403, 1988.

TORRES, I. L.; NARANJO-PIÑERA, E. J.; ANDRADE, D. M. G.; ALDÁN, E. C. Ecologia de Tapirus bairdii (Perissodactyla: Tapiridae) em La Reserva de La Biosfera El Triunfo (Polígono I), Chiapas, México. Acta Zoológica Mexicana, v. 20, n. 1, p. 1 - 21, 2004.

TROLLE, M.; NOSS, A. J.; CORDEIRO, J. L. P.; OLIVEIRA, L. F. B. Brazilian tapir density in the Pantanal: a comparison of systematic camera trapping and line transect surveys. Biotropica, v. 40, p. 211 - 217, 2007.

VIDOLIN, G. P. Ecologia, manejo e conservação do queixada Tayassu pecari no Parque Nacional das Emas e em propriedades rurais de seu entorno. 129 p. Tese (Doutorado em Conservação da Natureza) - Universidade Federal do Paraná, Curitiba, 2008.

ZIMMERMAN, B.; PERES, C. A.; MALCOLM, J. R.; TURNER, T. Conservation and development alliances with the Kayapó of south-eastern Amazonia, a tropical forest indigenous people. Environmental Conservation, v. 28, n. 1, p. 10 - 22, 2001.

ZOLLNER, P. A.; LIMA, S. L. Illumination and the perception of remote habitat patches by white-footed mice. Animal Behaviour, v. 58, p. 489 - 500, 1999. 